\title{
Noncommutative Instantons and Solitons
}

\section{Olaf Lechtenfeld*}

Institut für Theoretische Physik, Universität Hannover

E-mail: 'Iechtenf@itp.uni-hannover. de

ABSTRACT: I explain how to construct noncommutative BPS configurations in four and lower dimensions by solving linear matrix equations. Examples are instantons in $D=4$ Yang-Mills, monopoles in $D=3$ Yang-Mills-Higgs, and (moving) solitons in $D=2+1$ YangMills-Higgs. Some emphasis is on the latter as a showcase for the dressing method.

\section{Self-duality and BPS equations}

In this talk I shall present a powerful method for and results of constructing classical field configurations with finite action or energy in four-dimensional noncommutative gauge theory and its lower-dimensional descendants:

$$
\begin{aligned}
& D=4+0 \text { instantons } \longrightarrow \quad D=3+0 \text { monopoles } \\
& D=2+2 \quad \text { "waves" } \longrightarrow \quad D=2+1 \quad \text { solitons }
\end{aligned}
$$

I am setting up the formalism in such a way that it is completely transparent to the (Moyaltype) noncommutative deformation. In other words, the noncommutative equations below differ from the commutative ones merely in the interpretation of the symbols or their product (stars are suppressed). This will be briefly explained in Section 6. The Yang-Mills field equations are implied by first-order (self-duality or BPS) equations:

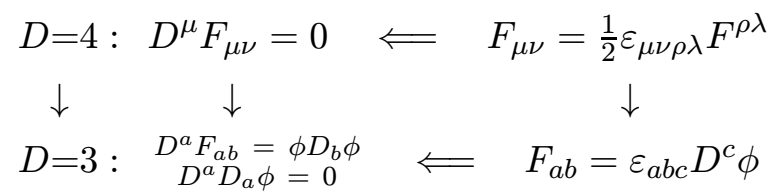

where $F$ and $\phi$ are $u(n)$ valued and Greek indices run from 1 to 4 while Latin ones stop at 3. In complex coordinates (note the signs!) $y=x^{1}+\mathrm{i} x^{2}$ and $z=x^{3} \mp \mathrm{i} x^{4}$ the self-duality equation $F=* F$ becomes

$$
\left[D_{y}, D_{z}\right]=0=\left[D_{\bar{y}}, D_{\bar{z}}\right] \quad \text { and } \quad\left[D_{y}, D_{\bar{y}}\right] \pm\left[D_{z}, D_{\bar{z}}\right]=0
$$

\footnotetext{
${ }^{*}$ Speaker.
} 
where the upper and lower signs belong to the signatures $(4,0)$ and $(2,2)$, respectively. Dimensional reduction to $D=3$ is accomplished via

$$
\partial_{4}=0, \quad A_{4}=\phi \quad \text { for } \quad D=3+0 \quad \text { or } \quad \partial_{3}=0, \quad A_{3}=\phi \quad \text { for } \quad D=2+1 \text {. }
$$

\section{Lax pair}

The three self-duality equations (1, (1)

$$
\left(D_{\bar{y}}-\lambda D_{z}\right) \Psi(x, \lambda)=0=\left(D_{\bar{z}} \pm \lambda D_{y}\right) \Psi(x, \lambda),
$$

where $\Psi(x, \lambda) \in \mathrm{U}(n)$ is a matrix function holomorphic in the spectral parameter $\lambda \in$ $\mathbb{C} P^{1} \simeq S^{2}$. From the auxiliary function $\Psi$ the gauge potential can be recovered via

$$
A_{\bar{y}}-\lambda A_{z}=\Psi\left(\partial_{\bar{y}}-\lambda \partial_{z}\right) \Psi^{-1} \quad \text { and } \quad A_{\bar{z}} \pm \lambda A_{y}=\Psi\left(\partial_{\bar{z}} \pm \lambda \partial_{y}\right) \Psi^{-1}
$$

In addition, antihermiticity of $A$ corresponds to a normalization condition for $\Psi$,

$$
A_{\mu}^{\dagger}=-A_{\mu} \quad \Longleftrightarrow \quad \Psi(x, \lambda) \Psi(x, \mp 1 / \bar{\lambda})^{\dagger}=\mathbf{1},
$$

which involves a reflection of $\lambda$ on the unit circle.

\section{Gauge fixing}

Out of the three self-duality equations (1) $(\overline{1}-\overline{5})$, the $(2,0)$ part $F_{y z}=0$ and the $(0,2)$ part $F_{\bar{y} \bar{z}}=0$ are solved by

$$
\begin{aligned}
A_{y} & =g^{-1} \partial_{y} g \\
A_{z} & =g^{-1} \partial_{z} g
\end{aligned} \quad \text { and } \quad \begin{aligned}
A_{\bar{y}} & =\tilde{g}^{-1} \partial_{\bar{y}} \tilde{g} \\
A_{\bar{z}} & =\tilde{g}^{-1} \partial_{\bar{z}} \tilde{g}
\end{aligned}
$$

for $g, \tilde{g} \in \mathrm{GL}(n, \mathbb{C})$, possibly with the restriction that

$$
A_{y}^{\dagger}=-A_{\bar{y}} \quad \text { and } \quad A_{z}^{\dagger}=-A_{\bar{z}} \quad \Longrightarrow \quad \tilde{g}=\left(g^{\dagger}\right)^{-1} .
$$

Allowing for $\Psi \in \mathrm{GL}(n, \mathbb{C})$ I may transform to the so-called hermitean gauge,

$$
\Psi \rightarrow \tilde{g} \Psi, \quad A_{\bar{y}} \rightarrow 0, \quad A_{\bar{z}} \rightarrow 0, \quad A_{y} \rightarrow h^{-1} \partial_{y} h, \quad A_{z} \rightarrow h^{-1} \partial_{z} h \quad,
$$

where $h=g \tilde{g}^{-1}=g g^{\dagger}=h^{\dagger}$. This gauge eliminates half of $A$, but the price to pay is that now $A_{y}^{\dagger} \neq-A_{\bar{y}}$ and $A_{z}^{\dagger} \neq-A_{\bar{z}}$ as well as

$$
\Psi(x, \lambda) \Psi(x, \mp 1 / \bar{\lambda})^{\dagger}=\tilde{g} g^{-1}=h^{-1} \neq 1 .
$$

The remaining $(1,1)$ part of the self-duality equations (1) 1.51 produces a second-order equation for the prepotential $h$ :

$$
F_{y \bar{y}} \pm F_{z \bar{z}}=0 \quad \Longrightarrow \quad \partial_{\bar{y}}\left(h^{-1} \partial_{y} h\right) \pm \partial_{\bar{z}}\left(h^{-1} \partial_{z} h\right)=0 \quad .
$$

In the hermitean gauge the linear system $\left(\overline{2} . \overline{2} \bar{l}_{i}\right)$ reads

$$
\left(\partial_{\bar{y}}-\lambda \partial_{z}\right) \Psi=\lambda A_{z} \Psi \quad \text { and } \quad\left(\partial_{\bar{z}} \pm \lambda \partial_{y}\right) \Psi=\mp \lambda A_{y} \Psi
$$

From its solution it is, in principle, always possible to retrieve an antihermitean gauge potential by an appropriate gauge transformation. 


\section{Dressing method}

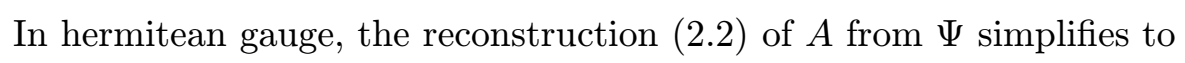

$$
A_{z}=\Psi(\lambda)\left(\partial_{z}-\frac{1}{\lambda} \partial_{\bar{y}}\right) \Psi(\lambda)^{-1} \quad \text { and } \quad A_{y}=\Psi(\lambda)\left(\partial_{y} \pm \frac{1}{\lambda} \partial_{\bar{z}}\right) \Psi(\lambda)^{-1}
$$

where $\Psi$ is subject to

$$
h^{-1}=\Psi(\lambda) \Psi(\mp 1 / \bar{\lambda})^{\dagger}
$$

Since $\lambda \in \mathbb{C} P^{1}$ a nonconstant matrix function $\Psi(\lambda)$ cannot be globally holomorphic. Hence, it must have poles at $\lambda=\mu_{k}, \quad k=1, \ldots, m$. The power of holomorphy then enables us to find $\Psi$ without knowing $A$, just by fixing its pole structure!

The dressing method [i] builds up $\Psi(x, \lambda)$ multiplicatively: $\Psi_{k \text { poles }}=\chi_{k} \cdot \Psi_{k-1}$ poles, employing the ansatz

$$
\chi_{k}(x, \lambda)=\mathbf{1}-\frac{\lambda\left(1 \pm \mu_{k} \bar{\mu}_{k}\right)}{\lambda-\mu_{k}} P_{k}(x)
$$

with moduli $\mu_{k}$ and matrices $P_{k}(x)$, and starting from the trivial seed solution $\Psi_{0}=\mathbf{1}$. An $m$-fold repetition of this dressing transformation yields

$$
\Psi_{m}(x, \lambda)=\prod_{k=1}^{m}\left(\mathbf{1}-\frac{\lambda\left(1 \pm \mu_{k} \bar{\mu}_{k}\right)}{\lambda-\mu_{k}} P_{k}(x)\right)=\mathbf{1}-\sum_{k=1}^{m} \frac{\lambda R_{k}(x)}{\lambda-\mu_{k}}
$$

if all moduli $\mu_{k}$ are mutually different.

\section{Single-pole ansatz}

A lot can be learned already from the simplest situation, namely $m=1$ (a single pole and moduli $\mu$ ):

$$
\Psi(x, \lambda)=\mathbf{1}-\frac{\lambda(1 \pm \mu \bar{\mu})}{\lambda-\mu} P(x)
$$

where the group-valued but $\lambda$-independent function $P$ is to be determined. It is crucial to observe that the left hand sides of $\left(\overline{4} \cdot \overline{1}^{\prime}\right)$ and $\left(\overline{4} \cdot \overline{2}^{\prime}\right)$ are $\lambda$-independent, implying that their right hand sides must have vanishing residues for the poles at $\lambda=\mu$ and $\lambda=\mp 1 / \bar{\mu}$. A short computation reveals the following:

$$
\begin{aligned}
& \left(\begin{array}{l}
1 \\
4
\end{array} 2^{\prime}\right) \quad \Longrightarrow \quad P^{2}=P=P^{\dagger} \quad \text { hermitean projector } \\
& \Longleftrightarrow \quad P=T \frac{1}{T^{\dagger} T} T^{\dagger} \quad n \times r(\text { ank }) \text { matrix } T(x) \\
& \left(\overline{1}_{\underline{4}} \overline{1}_{1}^{\prime}\right) \quad \Longrightarrow \quad P\left(\partial_{\bar{y}}-\mu \partial_{z}\right) P=0=(\mathbf{1}-P)\left(\partial_{z} \pm \bar{\mu} \partial_{\bar{y}}\right) P \\
& P\left(\partial_{\bar{z}} \pm \mu \partial_{y}\right) P=0=(\mathbf{1}-P)\left(\partial_{y}-\bar{\mu} \partial_{\bar{z}}\right) P \\
& \Longleftrightarrow \quad(\mathbf{1}-P) L T=0 \quad \text { with } \quad L:=\left\{\begin{array}{l}
\partial_{z} \pm \bar{\mu} \partial_{\bar{y}} \\
\partial_{y}-\bar{\mu} \partial_{\bar{z}}
\end{array}\right. \\
& \Longleftrightarrow \quad L T=T \gamma \quad \text { for some } r \times r \text { matrix } \gamma \text {. }
\end{aligned}
$$


I conclude: Every collection $T(x)$ of $r$ simultaneous "eigenvectors" of the differential operators $L$ gives rise to a valid projector $P(x)$ which, in turn, yields a prepotential and a self-dual gauge connection:

$$
h^{-1}=\mathbf{1}-(1 \pm \mu \bar{\mu}) P \quad \text { and } \quad A_{z}=\frac{1 \pm \mu \bar{\mu}}{\mu} \partial_{\bar{y}} P \quad, \quad A_{y}=\mp \frac{1 \pm \mu \bar{\mu}}{\mu} \partial_{\bar{z}} P
$$

\section{Noncommutative deformation}

Up to now it seems that I have just reformulated rather old results. However, everything still makes sense if I understand all products of functions in the deformed sense, i.e.

$$
\begin{aligned}
(f g)(x) \quad \text { means } \quad(f \star g)(x) & =f(x) \exp \left\{\frac{\mathrm{i}}{2} \overleftarrow{\partial}_{\mu} \theta^{\mu \nu} \vec{\partial}_{\nu}\right\} g(x) \\
& =f(x) g(x)+\frac{\mathrm{i}}{2} \theta^{\mu \nu}\left(\partial_{\mu} f\right)(x)\left(\partial_{\nu} g\right)(x)+\ldots
\end{aligned}
$$

with $\theta^{\mu \nu}=-\theta^{\nu \mu}=$ constant in $D=4+0$ dimensions. The coordinate functions then obey the (star) commutation rule

$$
x^{\mu} \star x^{\nu}-x^{\nu} \star x^{\mu}=\mathrm{i} \theta^{\mu \nu}
$$

For a given noncommutativity matrix $\left(\theta^{\mu \nu}\right)$ I can choose an orthonormal basis in which

$$
\left(\theta^{\mu \nu}\right)=\left(\begin{array}{cccc}
0 & \theta & 0 & 0 \\
-\theta & 0 & 0 & 0 \\
0 & 0 & 0 & \theta^{\prime} \\
0 & 0 & -\theta^{\prime} & 0
\end{array}\right)
$$

In this talk I specialize to $\theta^{\prime}=\theta$ in $4+0$ dimensions (self-dual noncommutativity) while lower dimensions enforce $\theta^{\prime}=0$. For my choice of complex coordinates this implies that (note the asymmetry!)

$$
y \star \bar{y}-\bar{y} \star y=2 \theta=\bar{z} \star z-z \star \bar{z} .
$$

Via the Moyal-Weyl correspondence, this structure (the deformed function algebra) can be realized equivalently by an operator algebra with the usual (compositional) product,

$$
(f(y, \bar{y}, z, \bar{z}), \star) \simeq\left(F\left(a, a^{\dagger}, b^{\dagger}, b\right), \cdot\right) .
$$

The latter is generated by two sets $\left(a, a^{\dagger} ; b, b^{\dagger}\right)$ of oscillator annihilation and creation operators subject to the Heisenberg algebra

$$
\left[a, a^{\dagger}\right]=1=\left[b, b^{\dagger}\right]
$$

which can be represented on a Fock space $\mathcal{H}_{2}$. Putting $2 \theta=1$ for convenience, the MoyalWeyl map and its inverse operate as follows,

$$
F=\text { Weyl-order }\left[f\left(a, a^{\dagger}, b^{\dagger}, b\right)\right] \quad \text { and } \quad f=F_{\star}(y, \bar{y}, z, \bar{z}),
$$

where star multiplication is implied when writing out the Weyl symbol $F_{\star}$ in terms of the coordinates. It is also worth noting that

$$
\begin{gathered}
\partial_{y} f \simeq-\left[a^{\dagger}, F\right] \quad, \quad \partial_{\bar{y}} f \simeq[a, F] \quad, \quad \partial_{z} f \simeq[b, F] \quad, \quad \partial_{\bar{z}} f \simeq-\left[b^{\dagger}, F\right], \\
\text { and } \int \mathrm{d}^{4} x f(x)=(2 \pi \theta)^{2} \operatorname{tr}_{\mathcal{H}_{2}} F
\end{gathered}
$$

where the two-oscillator Fock space $\mathcal{H}_{2}$ is spanned by

$$
\left|m_{1}, m_{2}\right\rangle=\frac{1}{\sqrt{m_{1} ! m_{2} !}}\left(a^{\dagger}\right)^{m_{1}}\left(b^{\dagger}\right)^{m_{2}}|0,0\rangle \quad \text { with } \quad m_{1}, m_{2} \in \mathbb{N}_{0} \quad .
$$




\section{7. $\mathrm{D}=2+1$ : Solitons in noncommutative Yang-Mills-Higgs}

Starting from $D=2+2$ with signature $(++--)$ I put $\theta^{\prime}=0$ and

$$
A_{3}=\phi \quad, \quad \partial_{3}=0 \quad, \quad x^{4}=-t \quad, \quad y=x^{1}+\mathrm{i} x^{2}=\sqrt{2 \theta} a .
$$

Instead of using the hermitean gauge (see Section 3) it is more convenient in this situation to switch to the so-called unitary gauge. For the spectral parameter this entails a transformation from the unit disk to the upper half plane,

$$
\lambda \rightarrow \zeta=\mathrm{i} \frac{1+\lambda}{1-\lambda} \quad \text { so that } \quad 1 / \bar{\lambda} \rightarrow \bar{\zeta}
$$

This time, the two combinations $A_{t}-A_{2}$ and $A_{1}+\phi$ are gauged away, but now staying within $\mathrm{U}(n)$ so that the normalization condition remains

$$
A_{\mu}^{\dagger}=-A_{\mu} \quad \Longleftrightarrow \quad \Psi(x, \zeta) \Psi(x, \bar{\zeta})^{\dagger}=\mathbf{1}
$$

The linear system looks slightly different, and $\Psi$ produces $A$ via

$$
2 A_{1}=\Psi(\zeta)\left(\partial_{1}-\zeta\left(\partial_{t}-\partial_{2}\right)\right) \Psi(\zeta)^{-1} \quad \& \quad 2 A_{2}=\Psi(\zeta)\left(\left(\partial_{t}+\partial_{2}\right)-\zeta \partial_{1}\right) \Psi(\zeta)^{-1}
$$

Consequently, the single-pole ansatz has to be modified to

$$
\Psi\left(a, a^{\dagger}, t, \zeta\right)=\mathbf{1}+\frac{\mu-\bar{\mu}}{\zeta-\mu} P\left(a, a^{\dagger}, t\right)
$$

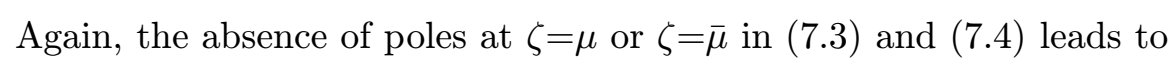

$$
\begin{aligned}
& \left(\overline{7}_{1} \cdot \overline{3}_{1}^{\prime}\right) \quad \Longrightarrow \quad P^{2}=P=P^{\dagger} \quad \Longleftrightarrow \quad P=T \frac{1}{T^{\dagger} T} T^{\dagger} \\
& (\overline{7}-\overline{4}) \quad \Longrightarrow \quad(\mathbf{1}-P) L P=0 \quad \Longleftrightarrow \quad L T=T \gamma
\end{aligned}
$$

where $L$ denotes the differential operators in $(\overline{7}, \overline{4})$ for $\zeta=\bar{\mu}$ and $\gamma$ is some $r \times r$ matrix.

In the noncommutative setup [2i] the $\left(x^{1}, x^{2}\right)$ coordinate dependence gets traded for operator valuedness while the time $t$ remains a parameter. The Heisenberg algebra $\left[a, a^{\dagger}\right]=$ 1 , when represented on $\mathcal{H}_{1}$ with basis $\left\{|m\rangle, m \in \mathbb{N}_{0}\right\}$, turns $A, \Psi$ and $P$ into semi-infinite $n \infty \times n \infty$ matrices acting on $\mathbb{C}^{n} \otimes \mathcal{H}_{1}$ for the gauge group $\mathrm{U}(n)$. The collection $T$ of $r$ column vectors is then seen as an $n \times r$ array of $\mathcal{H}_{1}$ kets:

$$
|T\rangle=\left(\left|T_{i}^{\ell}\right\rangle\right)_{i=1 \ldots n}^{\ell=1 \ldots r} \quad \Longrightarrow \quad P=|T\rangle \frac{1}{\langle T \mid T\rangle}\langle T|
$$

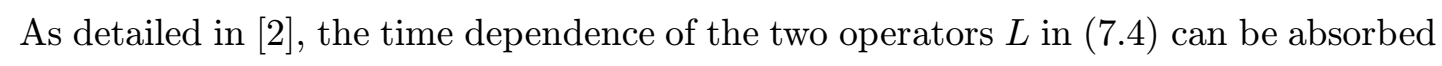
into an ISU $(1,1)$ coordinate transformation from $a$ to

$$
\begin{aligned}
& c=(\cosh \tau) a-\left(\mathrm{e}^{\mathrm{i} \vartheta} \sinh \tau\right) a^{\dagger}-\beta t=U(t) a U^{\dagger}(t) \\
& \text { with } \quad U(t)=\mathrm{e}^{\alpha a^{\dagger} a^{\dagger}-\bar{\alpha} a a} \mathrm{e}^{\left(\beta a^{\dagger}-\bar{\beta} a\right) t}
\end{aligned}
$$


where the parameters $\tau, \vartheta, \beta, \alpha$ are specific functions of $\mu$ only. These "moving-frame" coordinates are canonical, $\left[c, c^{\dagger}\right]=1$, and describe moving squeezed states

$$
|m\rangle_{t}=U(t)|m\rangle \quad \text { based on } \quad c|0\rangle_{t}=0 \quad .
$$

It turns out that both operators $L$ are proportional to $[c,$.$] , so that (\overline{7} . \overline{7} \cdot \bar{i})$ mutates to

$$
c\left|T_{i}^{\ell}\right\rangle=\left|T_{i}^{\ell^{\prime}}\right\rangle \gamma_{\ell^{\prime}}^{\ell} \quad \text { for some } r \times r \text { matrix } \quad \gamma=\left(\gamma_{\ell^{\prime}}^{\ell}\right) .
$$

This equation may be interpreted as a holomorphy condition. It is solved by any collection of states which spans a subspace of $\mathbb{C}^{n} \otimes \mathcal{H}_{1}$ invariant under the action of $c$. Each solution yields a time-dependent exact noncommutative $\mathrm{U}(n)$ soliton

$$
2 A_{1}=(\mu-\bar{\mu})\left(\partial_{t}-\partial_{2}\right) P \quad, \quad 2 A_{2}=(\mu-\bar{\mu}) \partial_{x} P
$$

with a topological charge $q$ and energy $E=8 \pi q \frac{\sqrt{1-v_{1}^{2}-v_{2}^{2}}}{1-v_{2}^{2}}$. Its energy density consists of up to $q$ lumps moving jointly in the $\left(x^{1}, x^{2}\right)$ plane with a constant velocity $\left(v_{1}, v_{2}\right)$ given by $\mu$. Having zero relative lump velocities these configurations cannot be considered as true multi-solitons. For $\mu=-\mathrm{i}$ one finds $\vec{v}=0$ and $c=a$, i.e. the static case $U(t)=1$.

Let us first look at solutions with finite rank $r<\infty$. An interesting class of solutions occurs for diagonal matrices $\gamma=\operatorname{diag}\left(\gamma_{1}, \ldots, \gamma_{r}\right)$, because then (72.1) decouples to

$$
c\left|T_{i}^{\ell}\right\rangle=\left|T_{i}^{\ell}\right\rangle \gamma_{\ell} \quad \forall i=1, \ldots, n \quad \text { and } \quad \ell=1, \ldots, r
$$

which is solved by coherent states based on the squeezed vacuum, as illustrated for $n=1$ :

$$
\left|T^{\ell}\right\rangle \sim \mathrm{e}^{\gamma_{\ell} c^{\dagger}}|0\rangle_{t}=U(t) \mathrm{e}^{\gamma_{\ell} a^{\dagger}}|0\rangle \sim U(t)\left|\gamma_{\ell}\right\rangle=\left|\gamma_{\ell}\right\rangle_{t} .
$$

Obviously, $\gamma_{\ell}$ simply gives the position of the $\ell$ th lump at $t=0$. For the simplest case, $r=1$ and $\vec{v}=0$, one finds

$$
P=|\gamma\rangle\langle\gamma|=\text { Weyl-order }\left[2 \mathrm{e}^{-2\left(a^{\dagger}-\bar{\gamma}\right)(a-\gamma)}\right] \quad \Longrightarrow \quad p(y)=2 \mathrm{e}^{-|y-\sqrt{2 \theta} \gamma|^{2} / \theta}
$$

which becomes singular in the commutative limit $\theta \rightarrow 0$. Any (abelian as well as non-abelian) finite-rank solution $|T\rangle$ has topological charge $q=r$ and can be built from such coherent states. Moreover, $|T\rangle$ is always unitarily related to the standard choice $(|0\rangle,|1\rangle, \ldots|r-1\rangle)$ belonging to $(\gamma)^{r}=0$. In this sense, all finite-rank solutions have abelian character, as exemplified here for $n=2, r=2$ at $\vec{v}=0$ :

$$
\left(\begin{array}{cc}
|0\rangle & |1\rangle \\
0 & 0
\end{array}\right)=\left(\begin{array}{cc}
|0\rangle\langle 0| & S^{\dagger} \\
S & 0
\end{array}\right)\left(\begin{array}{cc}
|0\rangle & 0 \\
0 & |0\rangle
\end{array}\right)
$$

where $S=\frac{1}{\sqrt{a a^{\dagger}}} a:|m\rangle \rightarrow|m-1\rangle$ is the shift operator.

Infinite-rank solutions can also feature finite topological charge if they are truely nonabelian. As a static $\mathrm{U}(2)$ example with $r=1+\infty$ but $q=1$ consider

$$
|T\rangle=\left(\begin{array}{c}
|0\rangle\langle 0| \\
S
\end{array}\right)(|0\rangle|1\rangle|2\rangle|3\rangle \ldots)=\left(\begin{array}{ccccc}
|0\rangle & 0 & 0 & 0 & \ldots \\
0 & |0\rangle & |1\rangle & |2\rangle & \ldots
\end{array}\right)
$$


which yields $P=|0\rangle\langle 0| \oplus 1 \quad$ on $\quad \mathcal{H}_{1} \oplus \mathcal{H}_{1}$. Using $\quad S|0\rangle=0 \quad$ and $\quad S S^{\dagger}=1$ but $S^{\dagger} S=1-|0\rangle\langle 0|$, the unitary transformation

$$
\left(\begin{array}{cc}
\frac{\epsilon}{K}|0\rangle\langle 0|-\frac{\sqrt{a^{\dagger} a}}{K} & \frac{\epsilon}{K} S^{\dagger} \\
S \frac{\bar{\epsilon}}{K} & S \frac{\sqrt{a^{\dagger} a}}{K} S^{\dagger}
\end{array}\right)\left(\begin{array}{c}
|0\rangle\langle 0| \\
S
\end{array}\right)=\left(\begin{array}{c}
\epsilon \\
a
\end{array}\right) \frac{1}{K}
$$

with $K=\sqrt{a^{\dagger} a+\bar{\epsilon} \epsilon}$ and parametrized by $\epsilon \in \mathbb{C}$ clearly maps

$$
|T\rangle \rightarrow|T(\epsilon)\rangle=|\mathcal{T}\rangle \frac{1}{\sqrt{\langle\mathcal{T} \mid \mathcal{T}\rangle}} \quad \text { with } \quad|\mathcal{T}\rangle=\left(\begin{array}{l}
\epsilon \\
a
\end{array}\right)(|0\rangle|1\rangle|2\rangle \ldots)
$$

Since $\lim _{\epsilon \rightarrow 0}|T(\epsilon)\rangle=|T\rangle$ the parameter $\epsilon$ may be regarded as a regulator. The complete basis of kets drops out when building the projector, and so the choice of $|\mathcal{T}\rangle$ is equivalent to taking

$$
T=\widehat{\mathcal{T}} \equiv\left(\begin{array}{c}
\epsilon \\
a
\end{array}\right) \quad \Longrightarrow \quad P=\left(\begin{array}{cc}
\frac{\epsilon \bar{\epsilon}}{K^{2}} & \frac{\epsilon}{K^{2}} a^{\dagger} \\
a \frac{\bar{\epsilon}}{K^{2}} & a \frac{1}{K^{2}} a^{\dagger}
\end{array}\right)
$$

Quite generally, for $|T\rangle=\widehat{T}(|0\rangle|1\rangle|2\rangle \ldots)$ with $\widehat{T}$ being an $n \times r^{\prime}$ array of operators, the condition $(\overline{17} \cdot \overline{1} \overline{2})$ translates to

$$
[c, \widehat{T}]=\widehat{T} \widehat{\gamma} \quad \text { for some } r^{\prime} \times r^{\prime} \text { array } \widehat{\gamma} \text { of operators } .
$$

In case $\widehat{\gamma}=\operatorname{diag}\left(\gamma_{1}, \ldots, \gamma_{r^{\prime}}\right)$ with c-numbers $\gamma_{\ell}$ the solution takes the simple form

$$
\widehat{T}_{i}^{\ell}=\mathrm{e}^{\gamma_{\ell} c^{\dagger}} f_{i}^{\ell}(c)
$$

with arbitrary functions $f_{i}{ }^{\ell}$ of $c$ only. The corresponding projectors have infinite rank in $\mathbb{C}^{n} \otimes \mathcal{H}_{1}$ but the topological charge (and the energy) is determined by the degrees of $f_{i}{ }^{\ell}$ if the latter are polynomial.

These nonabelian solutions possess a regular $\theta \rightarrow 0$ limit where they coincide with the known commutative solutions, which live in the Grassmannian $\operatorname{Gr}\left(n, r^{\prime}\right)=\frac{\mathrm{U}(n)}{\mathrm{U}\left(r^{\prime}\right) \times \mathrm{U}\left(n-r^{\prime}\right)}$. Formally, their noncommutative deformations are elements of $\operatorname{Gr}\left(n \infty, r=r^{\prime} \infty+q\right)$, and the abelian solitons are included at $\left(n=1, r^{\prime}=0\right)$.

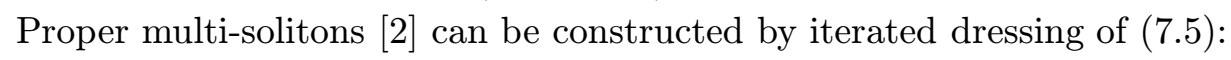

$$
\Psi_{m}\left(a, a^{\dagger}, t, \zeta\right)=\prod_{k=1}^{m}\left(\mathbf{1}-\frac{\mu_{k}-\bar{\mu}_{k}}{\zeta-\mu_{k}} P_{k}\left(a, a^{\dagger}, t\right)\right)=\mathbf{1}-\sum_{k=1}^{m} \frac{R_{k}\left(a, a^{\dagger}, t\right)}{\zeta-\mu_{k}}
$$

if all $\mu_{k}$ are mutually different. Repeating the previous analysis of the pole structure yields $P_{k}$ and $R_{k}$ in terms of $m$ moduli $\mu_{k}$ and ket matrices $|T\rangle_{k}$ subject to

$$
\left(\mathbf{1}_{n} \otimes c_{k}\right)|T\rangle_{k}=|T\rangle_{k} \cdot \gamma_{k} \quad \text { where } \quad c_{k}=U_{k}(t) a U_{k}^{\dagger}(t)
$$

is the "moving-frame" coordinate derived from $\mu_{k}$. In this fashion one arrives at $m$ copies of the single-pole solution; the corresponding (clusters of) lumps, however, move at mutually 
different velocities $\vec{v}_{k}$ ! Their topological charges and energies are simply additive. I should stress that the time dependence of these configurations is exact and not just valid in the adiabatic regime.

In this situation the question of scattering immediately emerges. An analysis of the asymptotic behavior shows that the ansatz $(\overline{7} .24)$ can only lead to no-scattering solutions, i.e. the lumps do not disturb one another. However, a slight generalization allowing for coinciding poles in $(7.24)$ produces nonabelian multi-solitons which scatter at angles $\vartheta=\pi / \ell$ as well as abelian breather-type configurations [3יin]

\section{8. $\mathrm{D}=4+0$ : Instantons in noncommutative Yang-Mills}

I will construct a self-dual finite-action U(2) configuration on a self-dual noncommutativity background. Not fixing the gauge, the basic relations are $(\overline{2} \cdot 2 \cdot \overline{2})$ and $(\overline{2} \cdot \overline{3})$. A one-instanton ansatz for $\Psi$ with poles at $\lambda=0$ and $\lambda=\infty$ reads $\left[\begin{array}{l}A \\ 0\end{array}\right.$,

$$
\Psi(x, \lambda)=G(x)\left(H(x)+\lambda S(x)^{\dagger}+\frac{1}{\lambda} S(x)\right) .
$$

On this I impose the following restrictions:

$$
\begin{gathered}
G=\left(\begin{array}{cc}
g_{-} & 0 \\
0 & g_{+}
\end{array}\right)=G^{\dagger} \quad, \quad H=\left(\begin{array}{cc}
h_{-} & 0 \\
0 & h_{+}
\end{array}\right)=H^{\dagger}, \\
{[G, H]=[G, S]=[H, S]=0 .}
\end{gathered}
$$

Inserting the above into $(\overline{2} \cdot \overline{2} \cdot \overline{2})$ and $(\overline{2} \cdot \overline{2} \cdot \overline{3})$ and separating different powers of $\lambda$ produces

$$
\begin{aligned}
\left(\overline{2} .3_{1}^{\prime}\right) \Longrightarrow & S^{2}=0, \quad G^{2} H^{2}=1+G^{2}\left\{S, S^{\dagger}\right\}, \\
\left(\overline{2} . \overline{2}_{1}^{i}\right) \Longrightarrow & S \partial_{\bar{y}} S=0=S \partial_{\bar{z}} S, \\
& H \partial_{\bar{y}} S-S \partial_{\bar{y}} H-S \partial_{z} S=0, \\
& H \partial_{\bar{z}} S-S \partial_{\bar{z}} H+S \partial_{y} S=0 .
\end{aligned}
$$

A convenient choice for $S$ is

$$
S=\left(\begin{array}{cc}
z \frac{1}{f(r)} y & z \frac{-1}{f(r)} z \\
y \frac{1}{f(r)} y & y \frac{-1}{f(r)} z
\end{array}\right) \quad \text { with } \quad r^{2}:=\bar{y} y+\bar{z} z=2 \theta\left(a^{\dagger} a+b b^{\dagger}\right)
$$

Then ( $\left(\overline{8} .3_{1}^{\prime}\right)$ is solved by $g_{ \pm}(x)=g\left(r^{2} \pm 2 \theta\right)$ and $h_{ \pm}(x)=h\left(r^{2} \pm 2 \theta\right)$, and furthermore

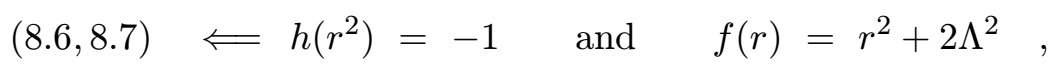

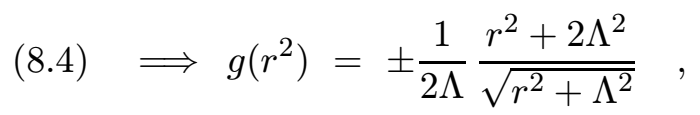

with some real parameter $\Lambda$. 
Putting it all together I arrive at

$$
\Psi=\frac{1}{2 \Lambda}\left(\begin{array}{cc}
\frac{1}{\sqrt{r^{2}+\Lambda^{2}-2 \theta}} & 0 \\
0 & \frac{1}{\sqrt{r^{2}+\Lambda^{2}+2 \theta}}
\end{array}\right)\left(\begin{array}{cc}
r^{2}+2 \Lambda^{2}-2 \theta-\lambda \bar{y} \bar{z}-\frac{y z}{\lambda} & -\lambda \bar{y}^{2}+\frac{z^{2}}{\lambda} \\
\lambda \bar{z}^{2}-\frac{y^{2}}{\lambda} & r^{2}+2 \Lambda^{2}+2 \theta+\lambda \bar{y} \bar{z}+\frac{y z}{\lambda}
\end{array}\right)
$$

which finally yields the noncommutative BPST instanton (see also [태] $)$ :

$$
\begin{aligned}
& A_{y}=\left(\begin{array}{cc}
-\frac{\bar{y}}{2 \theta}\left(\sqrt{\frac{r^{2}+\Lambda^{2}-2 \theta}{r^{2}+\Lambda^{2}}}-1\right) & 0 \\
-\bar{z} \frac{1}{\sqrt{r^{2}+\Lambda^{2}} \sqrt{r^{2}+\Lambda^{2}-2 \theta}} & -\frac{\bar{y}}{2 \theta}\left(\sqrt{\frac{r^{2}+\Lambda^{2}+4 \theta}{r^{2}+\Lambda^{2}+2 \theta}}-1\right)
\end{array}\right) \\
& A_{z}=\left(\begin{array}{cc}
\left(\sqrt{\frac{r^{2}+\Lambda^{2}-2 \theta}{r^{2}+\Lambda^{2}}}-1\right) \frac{\bar{z}}{2 \theta} & -\frac{1}{\sqrt{r^{2}+\Lambda^{2}} \sqrt{r^{2}+\Lambda^{2}-2 \theta}} \bar{y} \\
0 & \left(\sqrt{\frac{r^{2}+\Lambda^{2}+4 \theta}{r^{2}+\Lambda^{2}+2 \theta}}-1\right) \frac{\bar{z}}{2 \theta}
\end{array}\right) .
\end{aligned}
$$

\section{9. $\mathrm{D}=3+0$ : Monopoles in noncommutative Yang-Mills-Higgs}

Beginning from $D=4+0$ I set $\theta^{\prime}=0$ and

$$
A_{4}=\phi \quad \text { and } \quad \partial_{4}=0 \quad \Leftrightarrow \quad \partial_{z}-\partial_{\bar{z}}=0 \quad \text { and } \quad D \phi=* F
$$

One may again try to apply the dressing method, but it turns out that this situation is more amenable to the (related) splitting method, which reformulates the linear system (2.1. a parametric Riemann-Hilbert problem [i, $]$. Lacking the time to explain this approach in any detail I will only sketch the salient features for the monopole case [īin].

In all methods, the BPS equation gets reduced to a linear (differential) equation for an auxiliary object, e.g. for $T$ in ( $(\overline{7}-\overline{1})$ or $(7.12)$, which restricts the coordinate dependence of this quantity. Within the splitting approach, it is a matrix-valued so-called transition function $f_{+-}(x, \lambda)$ which in $D=3$ depends on the coordinates $x=\left(x^{1}, x^{2}, x^{3}\right)$ only holomorphically through the combination (remember $y=x^{1}+\mathrm{i} x^{2}$ and $[y, \bar{y}]=1$ )

$$
w(\lambda)=2 x^{3}+\lambda \bar{y}-\lambda^{-1} y
$$

The Riemann-Hilbert task of factorizing

$$
f_{+-}(x, \lambda)=\Psi_{+}^{-1}\left(x, x^{4}, \lambda\right) \Psi_{-}\left(x, x^{4}, \lambda\right) \quad \text { with } \quad \Psi_{ \pm}(\lambda) \text { holomorphic for }|\lambda|>1
$$

requires the multiplicative and additive decompositions

$$
w=\frac{1}{\lambda}\left(y \xi^{-1}+\lambda \xi\right)\left(\lambda \xi^{-1} \bar{y}-\xi\right)=(z+\lambda \bar{y})-\left(\lambda^{-1} y-\bar{z}\right)=u-v
$$

where $\xi$ was computed in $[\overline{9} \mid \overline{9}]$ and obeys $\xi^{2} \stackrel{\theta \rightarrow 0}{\longrightarrow} r-x^{3}$ with $r^{2}=\bar{y} y+x^{3} x^{3}$. 
In case of $u(2)$ monopoles, the simplest ansatz for the $2 \times 2$ matrix $f_{+-}$contains a function $\rho$ which for the BPS monopole can be reduced to the Weyl-ordered expression

$$
\begin{aligned}
\rho & =\mathrm{e}^{u} w^{-1} \mathrm{e}^{u}-\mathrm{e}^{v} w^{-1} \mathrm{e}^{v} \\
& =\mathrm{e}^{-2 \mathrm{i} x^{4}} \int_{-1}^{+1} \mathrm{~d} t \mathrm{e}^{2 t x^{3}} \mathrm{e}^{\lambda(1+t) \bar{y}+\lambda^{-1}(1-t) y} \\
& =\mathrm{e}^{-2 \mathrm{i} x^{4}} \sum_{m \in \mathbb{Z}} \rho_{m} \lambda^{m} \\
\text { where } \quad \rho_{0} & =\sinh (2 R) / R \quad \text { with } \quad R=x^{3}+\xi \xi \stackrel{\theta \rightarrow 0}{\longrightarrow} r \\
\rho_{ \pm 1} & =\operatorname{explicitly~known~in~terms~of~}\left(y, \bar{y}, x^{3}, R\right) .
\end{aligned}
$$

The gauge potential is entirely expressed in terms of $\rho_{0}$ via [is]

$$
\begin{aligned}
A_{i}= & \varepsilon_{i j k} \\
& \frac{\sigma_{k}}{2 \mathrm{i}}\left(\rho_{0}^{+\frac{1}{2}} \partial_{j} \rho_{0}^{-\frac{1}{2}}-\rho_{0}^{-\frac{1}{2}} \partial_{j} \rho_{0}^{+\frac{1}{2}}\right) \\
& +\frac{\mathbf{1}_{2}}{2}\left(\rho_{0}^{-\frac{1}{2}} \partial_{i} \rho_{0}^{+\frac{1}{2}}+\rho_{0}^{+\frac{1}{2}} \partial_{i} \rho_{0}^{-\frac{1}{2}}\right)+\sigma_{i}, \\
\phi \equiv A_{4}= & \frac{\sigma_{i}}{2 \mathrm{i}}\left(\rho_{0}^{+\frac{1}{2}} \partial_{i} \rho_{0}^{-\frac{1}{2}}-\rho_{0}^{-\frac{1}{2}} \partial_{i} \rho_{0}^{+\frac{1}{2}}\right) .
\end{aligned}
$$

The expression for $A_{i}$ is not antihermitean and was not expected to be because I did not impose a reality condition on the factorization on $f_{+-}$. However, it is possible to pass to an antihermitean configuration via a nonunitary gauge transformation generated by

$$
g=\left[\left.\Psi_{+}(\lambda) \Psi_{-}^{\dagger}(-1 / \bar{\lambda})\right|_{\lambda=0}\right]^{1 / 2} .
$$

Unfortunately, the matrix $g^{2}$ is quite complicated and involves $\rho_{ \pm 1}$ as well [8] the commutative limit reproduces the familiar result:

$$
A_{i}=\varepsilon_{i j k} \frac{\sigma_{k}}{2 \mathrm{i}} \frac{x_{j}}{r}\left(\frac{1}{r}-2 \operatorname{coth}(2 r)\right)+\sigma_{i} \quad \stackrel{g=\mathrm{e}^{x^{i} \sigma_{i}}}{\longrightarrow} \quad \varepsilon_{i j k} \frac{\sigma_{k}}{2 \mathrm{i}} \frac{x_{j}}{r}\left(\frac{1}{r}-\frac{2}{\sinh (2 r)}\right) .
$$

\section{Other applications}

The methods outlined in this talk have also been applied successfully towards the construction and study of various other noncommutative field configurations (see for example [1]

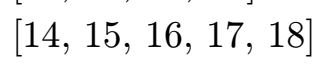

- Relations of noncommutative integrable models with open $N=2$ strings

- Seiberg-Witten monopole equations on $\mathbb{R}_{\theta}^{4}$, related with vortex-type equations on $\mathbb{R}_{\theta}^{2}$

- Donaldson-Uhlenbeck-Yau equations on $\mathbb{R}_{\theta}^{2 n} \times S^{2}$ give vortex-type equations on $\mathbb{R}_{\theta}^{2 n}$

\footnotetext{
${ }^{1}$ This is not a review. I apologize for my incomplete citation.
} 
- Moyal-deformed extended superspace and gauge theory thereon

- Open superstring field theory (à la Witten or Berkovits), which can be interpreted as an integrable infinite-dimensional noncommutative field theory

Numerous open problems remain to be tackled, such as finding nontrivial classical superstring configurations, analyzing the quantum fluctuations around our BPS solutions, or substantiate their D-brane interpretation.

\section{Acknowledgments}

I am grateful to my coworkers who helped bring this program to life.

\section{References}

[1] V.E. Zakharov and A.V. Mikhailov, Sov. Phys. JETP 47 (1978) 1017

[Zh. Eksp. Teor. Fiz. 74 (1978) 1953];

V.E. Zakharov and A.B. Shabat, Funct. Anal. Appl. 13 (1979) 166;

P. Forgács, Z. Horváth and L. Palla, Nucl. Phys. B 229 (1983) 77.

[2] O. Lechtenfeld and A.D. Popov, JHEP 0111 (2001) 040 [hep-th/0106213].

[3] O. Lechtenfeld and A.D. Popov, Phys. Lett. B 523 (2001) 178 [hep-th/0108118]; M. Wolf, JHEP 0206 (2002) 055 [hep-th/0204185].

[4] A.A. Belavin and V.E. Zakharov, Phys. Lett. B 73 (1978) 53.

[5] Z. Horváth, O. Lechtenfeld and M. Wolf, JHEP 0212 (2002) 060 [hep-th/0211041].

[6] K. Furuuchi, JHEP 0103 (2001) 033 [hep-th/0010119].

[7] R.S. Ward, Phys. Lett. A 61 (1977) 81;

R.S. Ward and R.O. Wells, Twistor geometry and field theory, Cambridge University Press, Cambridge, 1990.

[8] O. Lechtenfeld and A.D. Popov, Noncommutative monopoles and Riemann-Hilbert problems, hep-th/0306263.

[9] D.J. Gross and N.A. Nekrasov, JHEP 0007 (2000) 034 [hep-th/0005204]; JHEP 0103 (2001) 044 [hep-th/0010090].

[10] A. Dimakis and F. Müller-Hoissen,

A noncommutative version of the nonlinear Schrödinger equation, hep-th/0007015;

J. Phys. A 34 (2001) 9163 [nlin.si/0104071];

Extension of noncommutative soliton hierarchies, hep-th/0401142.

[11] M. Hamanaka and K. Toda, J. Phys. A 36 (2003) 11981 [hep-th/0301213];

Towards noncommutative integrable equations, hep-th/0309265;

M. Hamanaka, Commuting flows and conservation laws for noncommutative Lax hierarchies, hep-th/0311206.

[12] I. Cabrera-Carnero and M. Moriconi, Nucl. Phys. B 673 (2003) 437 [hep-th/0211193];

F. A. Schaposnik, Noncommutative solitons and instantons, hep-th/0310202;

M.T. Grisaru, L. Mazzanti, S. Penati and L. Tamassia, Some properties of the integrable noncommutative sine-Gordon system, hep-th/0310214. 
[13] N. Wang and M. Wadati, J. Phys. Soc. Japan 72 (2003) 1366; 1881; 3055.

[14] O. Lechtenfeld, A.D. Popov and B. Spendig, Phys. Lett. B 507 (2001) 317 [hep-th/0012200]; JHEP 0106 (2001) 011 [hep-th/0103196];

M. Ihl and S. Uhlmann, Int. J. Mod. Phys. A 18 (2003) 4889 [hep-th/0211263].

[15] A.D. Popov, A.G. Sergeev and M. Wolf, J. Math. Phys. 44 (2003) 4527 [hep-th/0304263].

[16] T.A. Ivanova and O. Lechtenfeld, Phys. Lett. B 567 (2003) 107 [hep-th/0305195]; O. Lechtenfeld, A.D. Popov and R.J. Szabo, JHEP 0312 (2003) 022 [hep-th/0310267].

[17] E. Ivanov, O. Lechtenfeld and B. Zupnik,

Nilpotent deformations of N=2 superspace, hep-th/0308012;

C. Sämann and M. Wolf, Constraint and super Yang-Mills equations on the deformed superspace $R_{\hbar}^{(4 \mid 16)}$, hep-th/0401147.

[18] O. Lechtenfeld, A.D. Popov and S. Uhlmann, Nucl. Phys. B 637 (2002) 119 [hep-th/0204155];

A. Kling, O. Lechtenfeld, A.D. Popov and S. Uhlmann, Phys. Lett. B 551 (2003) 193 [hep-th/0209186];

Fortsch. Phys. 51 (2003) 775 [hep-th/0212335];

A. Kling and S. Uhlmann, JHEP 0307 (2003) 061 [hep-th/0306254];

M. Ihl, A. Kling and S. Uhlmann,

String field theory projectors for fermions of integral weight, hep-th/0312314. 